\title{
Silo discharge: influence of the particle shape on the velocity profiles
}

\author{
Florencia Escudero $^{1}$, Marcela Camila Villagrán Olivares ${ }^{1}$, Rodolfo Uñac ${ }^{1}$, Ana María Vidales ${ }^{1}$, and Jesica Benito ${ }^{1, *}$ \\ ${ }^{1}$ INFAP, CONICET, Departamento de Física, Facultad de Ciencias Físico Matemáticas y Naturales, Universidad Nacional de San Luis, \\ Ejército de los Andes 950, D5700HHW, San Luis, Argentina.
}

\begin{abstract}
Experiments on the discharge of a silo with an inclined outlet are performed using flattened seeds in order to evaluate the validity of a previous theoretical formulation developed in our work group [1]. In that description, funnel flow regime is assumed to be based on a free fall parabolic arc. The shape of this arc is described with a parameter which is the only one involved in the flow rate formulation. An experimental analysis of the behavior of this parameter is carried out based on the geometry and shape of the grains within the silo. Also, video analysis of the silo discharge is performed in order to investigate the velocity profiles at the outlet of the hopper for these non-spherical particles. Experiments are contrasted with analytical predictions derived from the proposed formulation in order to assess and discuss its validity for the case of flattened particles.
\end{abstract}

\section{Introduction}

The study of granular flows within silos is a topic of great interest due to its influence on different industrial processes. In the food industry, the examples where silos and hoppers intervene in different processes are varied. Despite the fact that they have been used for centuries, there are still many unknowns regarding the behavior of the grains inside them, leading to economic losses and waste of raw granular material. Many industrial challenges involve an adequate design of silos determining the pressures exerted on the walls, the precise geometry for flow control and the characterization of the grains that will be stored [2-4].Therefore, it is highly relevant to characterize the granular flow within silos and hoppers, with especial attention on the behavior that typical food industry seeds have inside of them.

There are many experimental and numerical works dedicated to the description of granular flow using silos and hoppers [5-8]. However, the theoretical description of the phenomenon is still an open issue due to the complex dynamic present in the discharging process. Several assumptions have been made to develop theoretical and empirical correlations based on the concept that particles arches form and collapse at the outlet region and particles free fall just below these arches during the discharge [912]. At present, the phenomenological correlation stated by Beverloo remains still very robust and useful in practical problems $[9,13,14]$.

It is well known that the flow patterns found inside a silo can be classified in two main regimes separated by one transition region: mass flow (for low friction coefficients or sufficiently inclined hopper walls) and funnel flow (typical for flat-bottomed hoppers). Thus, the hopper angle determines the flow rate and has a clear effect in the veloc-

*e-mail: jbenito@unsl.edu.ar

A video is available at https://doi.org/10.48448/92jz-yx83 ity profiles at the outlet of the silo [15]. On the other hand, there are only few works dedicated to study the influence of the particle shape on the discharge rate $[13,16]$. Mellmann et al. [16], analyzed the influence of the shape of the particles, their flow properties and the outlet geometry on the mass flow during the discharge from a silo.

Recently, a study developed in our group (Villagrán et al. [1]) showed the influence of the angle of the hopper for different types of grains. Two theoretical models were developed for the silo discharge, depending on the type of flow regime. In the case of the funnel flow, it was assumed a free fall arc (in which the particles fall with a zero initial velocity) as in previous works. The flow rate equation was defined using a parameter, $\delta$, which is directly related to the parabolic shape of the arc (see Eq. 1 below). For the case of mass flow, the theoretical description was based on a continuous model. Both models were contrasted with experiments, resulting in a very good agreement.

The present work will be based in the same theoretical formulation developed by Villagrán et al. [1]. The parameter $\delta$ (involved in the proposed theoretical description) will be obtained experimentally for different seeds and its dependence with the shape of the grains will be analyzed. On the other hand, experimental velocity profiles at outlet of the hopper for non-spherical particles will be determined through video analysis during the silo discharging. Finally, these velocity profiles will be contrasted to predictions derived from the proposed formulation [1] in order to assess its validity for the case of flattened particles and different hopper angles.

\section{Experimental device and material characterization}

The experimental set-up is designed as a 2D silo made of glass as the one sketched in Figure 1. 


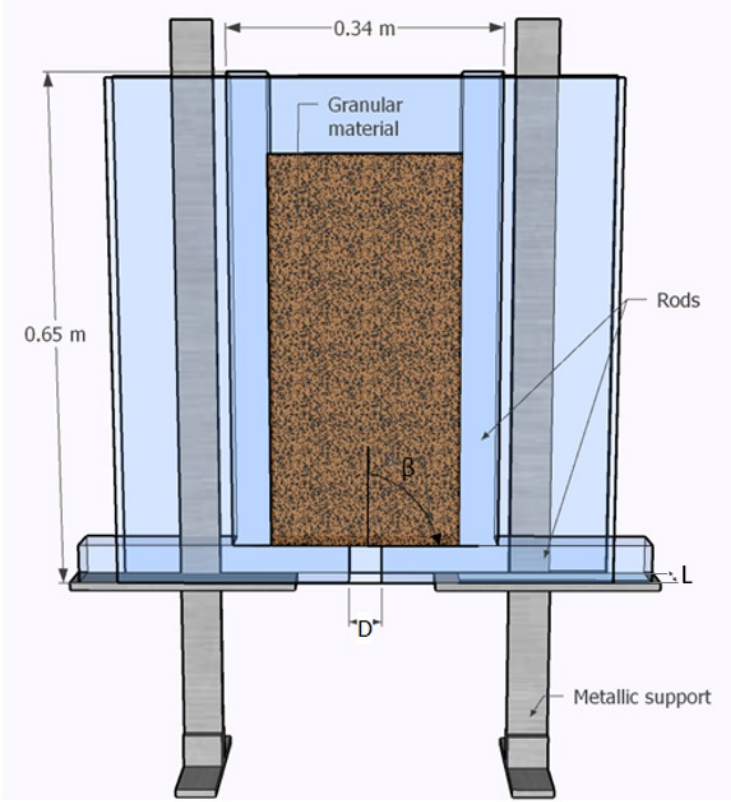

Figure 1. Schematic representation of the set-up used in the experiments.

It has four movable rods that allow varying both, the outlet opening $(D)$ and the tilt angle of the hopper $(\beta)$. The different parts of the device are held through presses and the complete structure is assembled on a support made out of aluminium. On top, a funnel is placed in order to load the silo with the grains (feeding system).

The discharge of the silo is performed inside a container placed on an electronic scale which is connected to a computer. Thus, from the recorded mass vs. time, the discharged flow rate $(W)$ can be easily obtained.

\subsection{Granular Material}

As mentioned in section 1, the aim of this work is to analyze the behavior of silos discharge in the case of nonspherical grains. For this purpose, lentil seeds are selected given that one of its characteristic lengths is very flattened. The grains are geometrically characterized using a sample of 50 lentils. The determination of the length $(a)$ and width $(b)$ is carried out by analyzing particle photographs through an image analysis software. The thickness $(c)$ is measured using a digital caliper. The equivalent diameter $d$ (which represents the diameter of a sphere with the same volume of the seed) is also calculated following the procedure explained in [1]. Finally, the bulk density of the granular material $\left(\rho_{a}\right)$ is determined through the weighing of a container with known volume into which the grains are gently poured from a constant height. The mass of the seeds divided by the known volume gives the value of $\rho_{a}$.

In order to corroborate the theoretical formulation for the discharged flow rate proposed in [1], a parameter $\delta$ has to be also determined for the lentils seeds (see next section 3). To perform an analysis of the dependence of this parameter on the shape of the particles, different grains used in a former work (Villagrán et al.[1]) will be also employed. Thus, in addition to lentils, Figure 2 shows grains of millet, sesame and canary seed.

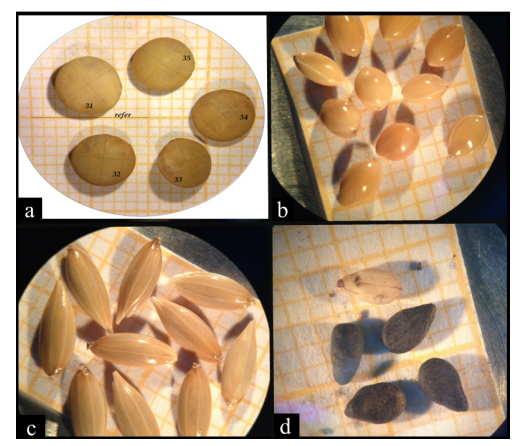

Figure 2. Seeds: a) lentil, b) millet, c) canary seed and d) sesame

Table 1 resumes the measurements of the geometric characteristics (with errors less than 8\%) and bulk densities (with $1 \%$ error) for each kind of seed.

Table 1. Values for different parameters of the seeds used in experiments

\begin{tabular}{ccccc}
\hline & Millet & Canary seed & Sesame & Lentil \\
\hline$a(\mathrm{~mm})$ & 3.4 & 6.0 & 3.4 & 5.7 \\
\hline$b(\mathrm{~mm})$ & 2.3 & 2.2 & 2.1 & 5.4 \\
\hline$c(\mathrm{~mm})$ & 2.3 & 2.2 & 2.1 & 2.3 \\
\hline$d(\mathrm{~mm})$ & 2.7 & 3.1 & 2.5 & 4.1 \\
\hline$\rho_{a}\left(\mathrm{~kg} / \mathrm{m}^{3}\right)$ & 780 & 760 & 640 & 830 \\
\hline
\end{tabular}

\subsection{Experimental procedure}

Silo discharge experiments for different combinations of outlet opening $(D)$, hopper angle $(\beta)$ and seed are performed. The experimental flow rate $W$ obtained for each parameters selection corresponds to the average of 5 discharge tests. In all the experiments, the relative humidity is less than $55 \%$, ensuring that the influence of capillary bridges being negligible. On the one hand, experimental values of the flow rate $(W)$ as a function of outlet opening $(D)$ are used to determine the parameter $\delta$, which will be discussed in section 3.1. On the other hand, discharges for lentil grains for different hopper angles are also filmed with a fast camera in order to perform an analysis of the velocity profile at the outlet, presented in section 3.2.

\section{Results}

\subsection{Correlation between the grain shape and $\delta$}

As mentioned before, in the previous work of Villagrán et al. [1] two different equations for $W$, one for funnel flow and the other for mass flow, have been developed. For the case of the funnel flow regime, the flow discharging from the silo is given by eq. 1 :

$$
W=\frac{\pi}{4} \rho_{a} L \sqrt{2 g \delta}(D-d)^{3 / 2}
$$

where $\rho_{a}$ is the bulk density, $L$ the thickness of the silo, $D$ the outlet opening, $d$ the equivalent diameter of the grains 
and $\delta$ represents an arc shape coefficient, being the only parameter involved in the whole description of the flow dynamics. Eq. 1 is based on the assumption that the discharge is governed by a continuous mechanism of formation and destruction of arches. The shape of these arches is assumed to be parabolic, with height $h$. Thus, the arc shape coefficient is defined as: $\delta=\frac{h}{(D-d)}$. It is worth to mention that eq. 1 does not depend on the hopper angle $\beta$ since it is only valid for funnel flow regime, i.e., for angles larger than the internal dynamic angle. More information on $\delta$, as well as on the experimental validation of eq. 1 for millet, sesame and canary seeds can be found in [1].

The experimental calculation of $\delta$ for the 4 seeds is performed using eq. 1 and a series of silo discharges for different outlets apertures $D$, in a flat-bottom configuration $\left(\beta=90^{\circ}\right)$. This is, parameter $\delta$ is determined by fitting the discharge flow $W$ as a function of $(D-d)$ using eq. 1 . The reader can find more details of this procedure in [1].

In order to analyze the dependence of $\delta$ with the shape or geometry of the particles, a form factor, $F F$, is defined as the quotient between the largest and smallest dimension of the seed, i.e., $F F=a / c$, which corresponds to the particle aspect ratio. Table 2 presents the values of $\delta$ and $F F$ obtained for different seeds and Figure 3 shows shows the dependence of $\delta$ with $F F$.

Table 2. Values of the parameters $\delta$ and the form factor $F F$ for the seeds used in experiments

\begin{tabular}{ccccc}
\hline & Millet & Canary seed & Sesame & Lentil \\
\hline$\delta$ & 0.86 & 0.41 & 0.65 & 0.58 \\
\hline$F F$ & 1.5 & 2.8 & 1.6 & 2.5 \\
\hline
\end{tabular}

Results show that $\delta$, which is directly related to the geometry of the parabolic discharge arc (usually very difficult to determine experimentally) is correlated to the form factor $F F$ : the larger the ratio between the longest and smallest dimensions of the seed (greater $F F$ ) the more flattened the outlet of the parabolic discharge cavity is (smaller $\delta$ ). This behaviour is in agreement with the results of Börzsönyi et al. [17] in the sense that the shape of the dome formed at the outlet of the silo has a height that decreases for more elongated particles.

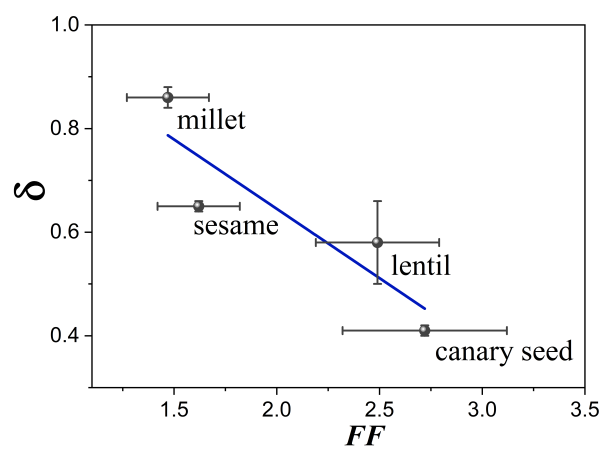

Figure 3. Shape arc parameter $\delta$ (obtained from the measurements in a flat-bottom configuration) as a function of the form factor $F F$ of the seeds.

\subsection{Analysis of the velocity profile at the silo outlet for flattened grains}

For funnel flow, in accordance with Villagrán et al. [1] proposal, the hypothesis of free fall from a height determined by the parabola of the exit arc should be fulfilled. Thus, the velocity can be obtained from:

$$
v_{\text {vertical }}=\sqrt{2 g f(x, y)}
$$

where $f(x, y)$ is

$$
f(x, y)=h\left(1-\left(\frac{2 x}{D-d}\right)^{2}\right) \text { for all } y
$$

Note that $x$ is the horizontal position along the outlet of the hopper with its zero taken at the middle of the silo. In eq. 3 the height $h$ is unknown, but it can be related to $\delta$, since $\delta=\frac{h}{(D-d)}$. For the case of lentil grains, using the experimental value for the arc shape parameter, $h=2.09$ $\mathrm{cm}$ and the expression for the velocity results:

$$
v_{\text {vertical }}=64\left(1-(0.557 x)^{2}\right)^{1 / 2} \mathrm{~cm} / \mathrm{s}
$$

The experimental velocity profiles are calculated through the processing of the videos obtained using a fast camera (approximately $1300 \mathrm{fr} / \mathrm{s}$ ). To achieve this objective, $3 \%$ of the lentils are painted in white. These painted grains are then identified in the space-time diagrams during their crossing through the outlet. The width of the outlet is divided into 12 sections and, from the analysis of these diagrams, the average velocity of all the marked seeds is calculated in each section. Figure 4 shows the experimental velocity profile for the case of a flat silo $\left(\beta=90^{\circ}\right)$ and the analytical prediction given by the equation above (eq. 4).

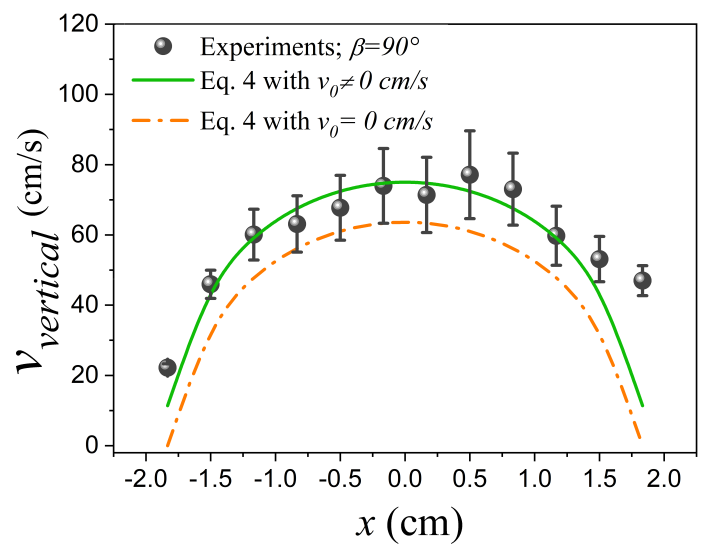

Figure 4. Velocity vs. $x$ at the hopper outlet for lentil seeds, $\beta=90^{\circ}$ and $D=4 \mathrm{~cm}$.

As it can be seen, the analytical prediction agrees quite well to the experimental results, showing that the parabolic arc hypothesis is correct and can describe properly the exit velocities for a flat silo and flattened particles. However, it is clear that the predicted values are systematically underestimated (see dotted line in Figure 4). This is due to the initial assumption that the grains reach the exit arc with a negligible velocity. This discrepancy observed suggests 
that initial velocity of the grains is not equal to zero. The same idea has been stated by Rubio-Largo et al. [18]. By means of experiments and numerical simulations they determined the existence of a singular region above the outlet where certain dynamical features decide the resulting velocity profile but, the idea of an arc where particles lose all their kinetic energy and start to fall freely under gravity was rejected.

Following this idea, if the average difference observed between the theoretical prediction (dotted line) and the experimental values is considered as the particle initial velocity $v_{0}$ at the outlet arc, this velocity can be added to the analytical results given by eq. 4. By doing this addition, the predicted velocity profile clearly agrees with experimental one (as shown by the solid line in Figure 4). This fact could indicates that particles initiate a free fall at the outlet arc but with a non-negligible initial velocity. This assumption still needs to be corroborated experimentally, through analysis of the videos above the outlet arc.

For the case of $\beta \neq 90^{\circ}$, as mentioned before, there is a change in the discharge regime. For angles close to $90^{\circ}$, the regime is expected to be more similar to a funnel flow and eq. 4 would be able to describe it adequately. Figure 5 shows the velocity profile for $\beta=60^{\circ}$ along with its theoretical prediction. The velocity distribution given by eq. 4 shows a good agreement with the experimental data. The angle used for this case is found precisely in the transition between both regimes and the change in the concavity of the distribution can also be appreciated. Further analysis of this case is on the way.

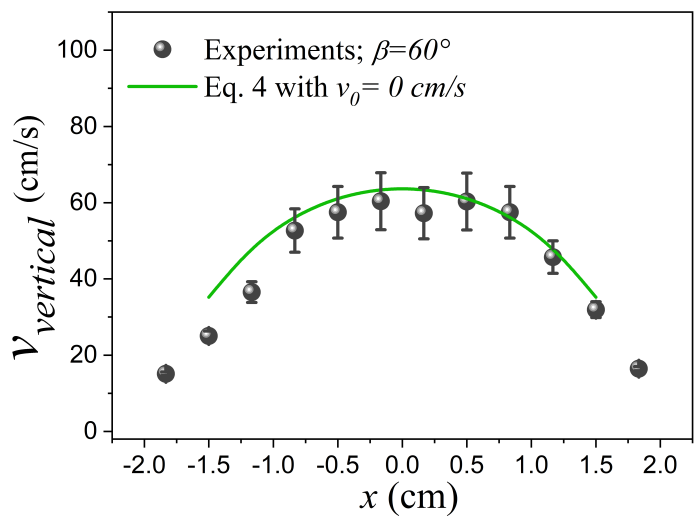

Figure 5. Velocity vs. $x$ at the hopper outlet for lentil seeds, $\beta=60^{\circ}$ and $D=4 \mathrm{~cm}$.

However, as expected, for smaller angles the validity of this formulation runs out and a more suitable formulation needs to be applied. In Villagrán et al. [1], a continuum model was developed and its validity for the case of flattened seeds will be tested in the near future. In this case, the shape of the particle and the way the seeds fall through the outlet should also be considered: grains falling with their minimum area of exposure to air (tip fall) or with the maximum exposure area (flat fall).

\section{Conclusion}

Silo discharging experiments were performed using flattened seeds, different hopper angles and outlet openings.
These experiments along with the analytical formulation for the funnel flow regime allowed the analysis of the parameter $\delta$ and its relationship with the shape of the grains. The larger the ratio between the longest and smallest dimensions of the seed, the smaller $\delta$, implying that the cavity for this outlet arc also reduces. On the other hand, experimental determination of velocity profiles were also performed for this kind of seeds through a video analysis. Comparison between the theoretical prediction and experiments was successful for large values of $\beta$, demonstrating that the proposed description is still valid for flattened grains. Future efforts will be dedicated to a full analysis of velocity profiles for smaller angles, taking properly into account the shape of the seeds.

Authors want to acknowledge to Universidad Nacional de San Luis and CONICET for financial support through respective projects PROICO 032718 and PIP $11220170100245 \mathrm{CO}$.

\section{References}

[1] M.C. Villagrán Olivares, J.G. Benito, R.O. Uñac, A.M. Vidales, Powder Technol. 336, 265-272 (2018)

[2] N. Job, A. Dardenne and J.-P. Pirard, J. Food Eng. 91(1), 118-125 (2009)

[3] J.J. Fitzpatrick, T. Iqbal, C. Delaney, T. Twomey, M.K. Keogh, J. Food Eng. 64(4), 435-444 (2004)

[4] C. Fürll and T. Hoffmann, Powder Technol. 235, 307311 (2013)

[5] C. Mankoc, A. Janda, R. Arévalo, J.M. Pastor, I. Zuriguel, A. Garcimartín and D. Maza, Granul. Matter 9, 407-414 (2007)

[6] A. Anand, J.S. Curtis, C.R. Wassgren, B.C. Hancock, W.R. Ketterhagen, Chem. Eng. Sci. 63, 5821-5830 (2008)

[7] J. Wu, J. Binbo, J. Chen, Y. Yang, Adv. Powder Technol. 20, 62-73 (2009)

[8] R.O. Uñac, O.A. Benegas, A.M. Vidales, I. Ippolito, Powder Technol. 225, 214-220 (2012)

[9] W.A. Beverloo, H.A. Leniger, J. van de Velde, Chem. Eng. Sci. 15, 260-269 (1961)

[10] R.L. Brown and J.C. Richards, Principles of Powder Mechanics (Pergamon Press, 1st Edition, 1970)

[11] A.J. Carleton, Powder Technol. 6, 91-96 (1972)

[12] I. Oldal, I. Keppler, B. Csizmadia, L. Fenyvesi, Adv. Powder Technol. 23, 290-297 (2012)

[13] C.A. Calderón, M.C. Villagrán Olivares, R.O. Uñac, A. M. Vidales, Powder Technol. 320, 43-50 (2017)

[14] S.D. Liu, Z.Y. Zhou, R.P. Zou, D. Pinson, A.B. Yu, Powder Technol. 253, 70-79 (2014)

[15] J.R. Darias, D. Gella, M.E. Fernandez, I. Zuriguel, D. Maza, Powder Technol. 366, 488-496 (2020)

[16] J. Mellmann, T. Hoffmann, C. Fürll, Powder Technol. 253, 46-52 (2014)

[17] T. Börzsönyi, E. Somfai, B. Szabó, S. Wegner, P. Mier, G. Rose, R. Stannarius, New J. Phys. 18, 093017 (2016)

[18] S. M. Rubio-Largo, A. Janda, D. Maza, I. Zuriguel and R. C. Hidalgo, Phys. Rev. Lett. 114, 238002 (2015) 